\title{
Le choix du mouvement : stratégie(s) communautaire(s) des Kazakhs-Mongols
}

The Choice of Movement: Kazakh-Mongols' Communitarian Strategies

El movimiento por la opción del movimiento: estrategia(s) comunitaria(s) de los Kazakos-Mongoles

Gaëlle Lacaze

\section{(2) OpenEdition}

Journals

Édition électronique

URL : https://journals.openedition.org/remi/5233

DOI : $10.4000 /$ remi.5233

ISSN : $1777-5418$

Éditeur

Université de Poitiers

Édition imprimée

Date de publication : 1 décembre 2010

Pagination : 169-180

ISBN : 978-2-911627-56-9

ISSN : 0765-0752

Référence électronique

Gaëlle Lacaze, «Le choix du mouvement : stratégie(s) communautaire(s) des Kazakhs-Mongols », Revue européenne des migrations internationales [En ligne], vol. 26 - n³ | 2010, mis en ligne le 01 décembre 2013, consulté le 15 avril 2022. URL : http://journals.openedition.org/remi/5233 ; DOI : https://doi.org/10.4000/remi.5233 


\section{Note de recherche}

\section{Le choix du mouvement : stratégie(s) communautaire(s) des Kazakhs-Mongols}

\section{Gaëlle LACAZE ${ }^{1}$}

T'Asie Intérieure offre un lieu de rencontre de différents peuples turco-

Lmongols. Plusieurs groupes ont ainsi construit des identités d'une grande complexité. Les Kazakhs de Mongolie constituent un exemple parmi d'autres de ces processus. Leur situation de population acculturée est atypique. Les Kazakhs forment un groupe « minoritaire » en Mongolie. Depuis 1991, ils sont invités à quitter la Mongolie pour revenir au Kazakhstan, leur « pays ancestral ». Ils y deviennent des Oralmandar (kz. : sing. Oralman, pl. Oralmandar), des « Rapatriés ». Compte tenu de la complexité des identités qu'ils développent, aujourd'hui, nous devons adopter des conventions terminologiques. Je distingue, dans cet article, les « Kazakhs de Mongolie » de ceux qui ont migré au Kazakhstan après 1990, les « Oralman mongols » et j'utilise l'expression « KazakhsMongols » pour dénommer sans les différencier les membres de ces deux communautés.

Cet article se fonde, entre autres, sur la récente monographie de Diener, One homeland or Two? The Nationalization et Transnationalization of Mongolia's Kazakhs (2009), qui étudie les situations contemporaines vécues par les Kazakhs-Mongols. L'intérêt de cette monographie réside avant tout dans l'originalité de sa démarche, aucun ouvrage n'ayant aujourd'hui été consacré aux Kazakhs-Mongols. En outre, cet ouvrage vient combler la rareté des informations concernant cette population autant dans les sources sur la Mongolie que dans celles sur le Kazakhstan ou dans les rapports des organismes internationaux.

Cet article se base également sur les matériaux que j'ai rassemblés lors de différentes enquêtes menées en Mongolie et au Kazakhstan. Parmi les quelque 465000 Oralman que compterait le Kazakhstan en 2005 (UNDP 2006 : 13), les Oralman mongols

1 Maître de conférences, Université de Strasbourg (détachée d'enseignement à l'Université de Picardie - Jules Verne); gaelle.lacaze@misha.fr 
sont les « laissés pour compte » des différentes études ${ }^{2}$. Lors de l'enquête de terrain que j'ai effectuée en 2002 au Kazakhstan, j'ai rencontré plusieurs représentants d'ONG kazakhes actifs auprès des Oralman, notamment des membres de l'association ASAR créée par des Kazakhs de Mongolie. Les chiffres qu'ils m'ont alors donnés incluent les Oralman venus en dehors du système de quotas. J'ai également récolté différents documents élaborés par les sections locales de l'OIM et du BIT et par divers organismes des Nations Unies.

Les destinées des Kazakhs-Mongols, qu'ils soient Kazakhs de Mongolie ou Oralman mongols, sont intrinsèquement liées aujourd'hui. Cette relation apparaît d'emblée dans l'estimation de leur nombre total et dans celui respectif à chaque communauté. Nous commencerons donc par éclaircir plusieurs questions concernant le dénombrement des Kazakhs-Mongols avant et après leurs migrations au Kazakhstan. Nous examinerons ensuite les groupes constitués, d'une part, par les Kazakhs de Mongolie et, d'autre part, par les « Oralman mongols » du Kazakhstan.

L'analyse des identités et des stratégies développées dans ces deux contextes illustre un choix implicite des Kazakhs-Mongols : parcourir les routes transnationales, se mouvoir le long de réseaux « ethniques » de socialisation et d'échange. Les «Oralman mongols » développent aujourd'hui des stratégies qui impliquent des mouvements à travers les sommets de l'Altaï, de larges réseaux de coopération et une conscience communautaire fondée sur une identité kazakhe complexe, politique, territoriale et ethnique. Je finirai par une brève note de recherche, présentée sous la forme d'un épilogue, concernant les migrations actuelles des Mongols de Mongolie.

\section{UNE ESTIMATION DIFFICILE}

En 2005, un rapport de 1'UNDP, Status of Oralmans in Kazakhstan estimait à 80000 le nombre de Kazakhs en Mongolie - soit moins de $4 \%$ de la population totale du pays - et à 71500 le nombre d'Oralman mongols au Kazakhstan (UNDP, 2006 : 8 , 13). Selon ces chiffres, l'UNDP évaluait donc à 151500 le nombre de KazakhsMongols. Or, selon les statistiques mongoles, en 1989, on comptait 120000 Kazakhs en Mongolie, soit 6,1\% de la population, dont plus de $60 \%$ résidaient « à la campagne » (Population of Mongolia, 1994 : 37-38). C'est également ce chiffre qui est retenu par Diener qui estime, en revanche, qu'au total 67000 Kazakhs de Mongolie auraient migré au Kazakhstan entre 1991 et 2005. Il reconnaît, par ailleurs et en accord avec les chiffres

2 Citons, par exemple, le très documenté rapport de la FIDH Kazakhstan/Kirghizstan : exploitation of migrants workers, protection denied to asylum seekers and refugees (2004). Ce rapport mentionne rarement les Oralman mongols alors qu'ils y constituent quantitativement la seconde population d'Oralman après les Ouzbeks, quant à eux régulièrement cités dans ce rapport. De même, les Oralman mongols sont peu évoqués dans le très documenté article de Mendikulova. Comment expliquer cette absence ? Les Oralman mongols se situent à la marge du processus de migration ethnique des Kazakhs ; ils représentent une catégorie interstitielle d'Oralman, liée au statut de leur pays d'origine. La Mongolie n'a pas fait partie de l'URSS, à la différence des pays de l'actuelle CEI d'où émigre un grand nombre d'Oralman. C'était néanmoins une République socialiste, à la différence des pays de provenance des Oralman non-russophones, la Chine faisant exception. 
de l'UNDP, qu'il demeure environ 80000 Kazakhs en Mongolie en 2005. Ce qui élève alors le nombre actuel de Kazakhs-Mongols à environ 147000 (Diener 2009 : 244). Or, les sources mongoles et internationales s'accordent à dire qu'en 2010, il reste plus de 100000 Kazakhs en Mongolie soit $5 \%$ de la population totale du pays. À partir de ces données et si l'on prend également en compte les « Oralman mongols », selon les chiffres Diener et ceux de l'UNDP, les Kazakhs-Mongols compteraient environ 170000 personnes entre la Mongolie et le Kazakhstan. L'estimation du nombre de Kazakhs-Mongols et, par conséquent, celle de leur répartition entre la Mongolie et le Kazakhstan pose donc différents problèmes.

Fin 1995, lors de sa visite en France, M. Dj. Enkhsaïkhan, secrétaire-général du Conseil de Sécurité nationale (Mongol Ulsijn ündesnij ajulgüj bajdlijn dzövlöl), instance dont le rôle est consultatif et qui réunit le président, le Premier ministre et le président du Parlement, reconnaissait que les Mongols ne disposaient «pas de chiffres sûrs concernant le nombre de Kazakhs de Mongolie installés au Kazakhstan. [...] Une partie d'entre eux revient, et les départs semblent diminuer »(Even, 1996). Combien de Kazakhs ont quitté la Mongolie ? Combien en reste-t-il ?

Les problèmes posés par le décompte des Kazakhs-Mongols ne sont pas irrésolubles. La différence entre le décompte de l'UNDP et les chiffres de Diener peut provenir des difficultés à recenser les populations d'Oralman, mobiles et flexibles. Ainsi, en 2004, l'UNDP (UNDP, 2006 : 10) estime que le nombre d'Oralman dépasse de $86 \%$ les quotas autorisés cette année-là. En outre, nous y reviendrons dans la dernière partie de cet article, il faut tenir compte des stratégies migratoires des Kazakhs-Mongols : les départs au Kazakhstan et les retours en Mongolie, dont l'importance est un fait acquis, ainsi que le développement de stratégies orientées vers la mobilité géographique et la flexibilité statutaire. Ces paramètres rendent effectivement difficile le calcul du nombre de KazakhsMongols, car un tiers d'entre eux participerait à plusieurs décomptes démographiques et relevés statistiques, à la fois, en Mongolie, en Russie, en Chine et au Kazakhstan.

\section{LES KAZAKHS DE MONGOLIE}

Au cours des années $1990^{3}$, l'appel à la migration ethnique du gouvernement kazakh, dans le cadre de la politique de rapatriement des « diasporas » kazakhes en vue de « re-kazakhiser » le Kazakhstan ${ }^{4}$, mise en place par le président kazakh N. Nazarbaev, souleva l'engouement des Kazakhs de Mongolie. Nous reviendrons sur les conséquences de cet appel à la migration ethnique dans la seconde partie de cet article. La ou les communauté(s) formée(s) par les Kazakhs de Mongolie ont alors connu une profonde reconfiguration démographique et sociale, interrogeant les identités qu'ils avaient précédemment construites, et qu'il nous faut maintenant décrire.

3 En 1990, les Kazakhs de Mongolie vivaient essentiellement dans la province occidentale de Bajanölgij et dans la région minière de Nalajh, près d'Ulaanbaatar.

4 En 1989, le Kazakhstan comptait seulement $40 \%$ de Kazakhs et $60 \%$ d'autres nationalités, essentiellement des Russes, mais également des Coréens, des Ouzbeks, des Kirghiz, etc. 
La Mongolie comme le Kazakhstan sont nés durant la période socialiste à travers l'enjeu du contrôle du territoire. Actuellement, ces États nations se caractérisent, entre autres, par l'émergence de courants nationalistes. Ainsi, les Kazakhs de Mongolie forment un groupe minoritaire dans un État-nation largement dominé par les «purs Mongols » (mg. : cever mongol), c'est-à-dire, les Mongols Halh. Ils développent aujourd'hui des processus identitaires inscrits dans ces sentiments nationalistes et se caractérisent en tant que Kazakhs-Mongols.

L'histoire de la « horde méridionale » (kz. : orta žuuz) kazakhe, regroupant entre autres les tribus Kereï et Naïman, majoritaires parmi les Kazakhs de Mongolie, s’imbrique à celle des peuples mongols dont ce sont des «voisins » historiquement proches ${ }^{5}$. La majorité des Kazakhs de Mongolie est issue de migrations « volontaires » effectuées entre 1860 et 1880 en provenance de Chine (Diener, 2009 : 264). En Mongolie, rares sont les Kazakhs directement chassés du Kazakhstan lors des purges de Staline ; peu d'entre eux ont migré pour fuir la collectivisation et les famines du XXe siècle. Le lien des Kazakhs de Mongolie aux régions de l'Altaï et aux populations mongoles qui y résident également se retrouve dans les dynamiques identitaires qu'ils ont développées au cours du XXe siècle et dans celles qu'ils déploient en réponse aux possibilités de rapatriement au Kazakhstan. Diener dans la monographie qu'il consacre aux Kazakhs de Mongolie s'interroge sur la nature de cette identité : est-elle « hybride ${ }^{6} »$ ou « diasporique $^{7} »$ ?

Plusieurs éléments tendent à montrer que l'identité des Kazakhs de Mongolie ne se réduit pas à une forme d'hybridité. En effet, les Kazakhs les plus mongolisés vivent dans les régions du centre du pays ; la plupart travaille dans le secteur minier et appartient aux classes sociales moyennes. Dans les campagnes de la province mongole de Bajan-ölgij, en revanche, les Kazakhs âgés parlent peu le mongol et, parmi les jeunes, beaucoup de ruraux n'en connaissent que des rudiments. Les contacts des Kazakhs avec la culture mongole n'entraînèrent donc pas leur acculturation profonde. Aujourd'hui encore, la situation des femmes kazakhes en Mongolie illustre le rigorisme de la culture kazakhe islamisée par opposition à la culture mongole bouddhisée, les femmes mongoles étant censées avoir des mœurs « libres ». Si l'on peut parler d'hybridation des Kazakhs de Mongolie, elle ne concerne donc que l'élite de cette communauté.

Contrairement à ce qu'avance Diener (2009 : 36-40), les Kazakhs ne semblent pas, non plus, développer une « identité diasporique ${ }^{8} »$. Cette conception identitaire

5 On retrouve ces tribus dans l'Histoire secrète des Mongols (Even, 1994), récit hagiographique de la dynastie gengiskhanide. En outre, ils jouent un rôle dans les conflits impliquant les Ö̈rats, leurs voisins mongols localisés en Djoungarie, dans le Xinjiang (Chine).

6 L'hybridité implique la notion d'une pureté originelle, état qui n'intervient jamais dans le cas des populations. Les géographes emploient cependant cette notion pour insister sur l'acculturation réussie d'un groupe diasporique dans un État d'accueil.

7 Selon Diener, la diaspora se définit par plusieurs éléments, dont le mythe du retour et la conception d'une « terre natale » versus « terre d'accueil » (2009: 9-10).

8 Diener invite, lui-même, à nuancer la notion de « diaspora » concernant les Kazakhs de Mongolie car ceux-ci se sont (re)territorialisés en Mongolie. Or, généralement, les groupes diasporiques apparaissent davantage comme « déterritorialisés » et « déplacés » (ibid. : 38). 
implique un processus de (dé)territorialisation. Ce processus n'est pas clair dans le cas de la communauté des Kazakhs de Mongolie. Il implique une redéfinition de la notion de « terre natale » (ibid. : 34). En effet, bien qu'arrivés à la suite de mouvements migratoires dans la province la plus occidentale de Mongolie, les Kazakhs de la " horde méridionale » sont historiquement localisés dans cette région montagneuse et semi-aride caractérisée par la présence de l'Altaï'. Ils pratiquent traditionnellement un élevage extensif, comparable à celui des Mongols de la région ${ }^{10}$. Leurs territoires de nomadisation les ont conduits à séjourner ponctuellement dans la partie orientale de l'Altaï, ne serait-ce que pour échapper aux $z u d^{11}$. La province de Bajan-ölgij ne se trouve pas considérée comme une terre d'accueil différente de la « terre natale » de référence des Kazakhs de Mongolie. Elle se situe, au contraire, dans son prolongement dans le contexte d'une notion de « territoire de nomadisation $»$.

En outre, les Kazakhs de Mongolie n'ont développé un « mythe du retour» au Kazakhstan qu'à la fin des années 1980, quand les mouvements nationalistes émergents ont rendu leur présence en Mongolie difficile et leur installation au Kazakhstan envisageable. Ils n'ont donc que récemment agrémenté leur(s) définition(s) identitaire(s) d'une dimension « diasporique ». Les Kazakhs ne sont pas les seuls « étrangers » en Mongolie où beaucoup de communautés cumulent une identité culturelle de " petit peuple ${ }^{12}$ » et une citoyenneté mongole. La construction de la RPM entraîna la hiérarchisation des peuples mongols, certains se trouvant relégués au rang de «minorité » (mg. : jastan) dans la « nation » (mg. : ündesten) mongole dominée par les Halh ${ }^{13}$. Ainsi, les Mongols Darhad jouissent-ils du même statut que les Kazakhs de Mongolie : ils sont une jastan dans la nation mongole. Pour les Darhad, en revanche, les références nationales et citoyennes relèvent du même registre culturel, la mongolité. Ce n'est pas le cas pour les Kazakhs qui gravitent entre deux cultures proches. Ces derniers n'appartiennent donc pas à la catégorie des « métis » (mg. : erlijz) qui désignent majoritairement les peuples mongols non Halh ou non-gengiskhanides (Bulac, 1998). Plus que d'autres jastan de Mongolie, les Kazakhs

9 Les Kazakhs de Mongolie sont majoritairement arrivés dans la province de Bajan-ölgij depuis la province du Xinjiang (Chine). Les groupes kazakhs qui y demeurent aujourd'hui se situent dans le nord de la province, essentiellement sur les sommets septentrionaux de l'Altaï.

10 Les troupeaux sont dominés par le cheval et comportent de nombreux chameaux. Les parcours de nomadisation alternaient, traditionnellement, entre les hauts pâturages d'hiver et les pâturages estivaux des vallées.

11 Les zud sont des intempéries caractérisées par leurs conséquences sur les troupeaux. Les zud «blancs » ou « noirs », avec trop ou trop peu de précipitation, que ce soient des zud d'été ou d'hiver, entraînent d'importantes pertes de bétail. Ce terme est commun aux langues mongoles et kazakhes.

12 En ex-URSS, un grand nombre de «petits peuples » (rs. : malenkie narody) connaît cette dualité identitaire entre citoyenneté et nationalité.

13 Ce processus a été analysé par Bulac (1998). Son analyse met en évidence, parfois de manière peu objective, le processus nationaliste de construction de la mongolité au cours des XIXe et XXe siècles. 
ont donc développé une sous-culture ${ }^{14}$ qui ne mélange pas les deux cultures de référence, mais préserve les éléments identitaires structurels de la culture nationale dans la culture dominante du pays d'accueil.

La sous-culture des Kazakhs de Mongolie ne correspond pas à une identité transnationale, mais bien comme Diener l'avance en conclusion de son ouvrage (2009: 336), à une série d'identités complexes alliant des appartenances ethniques et territoriales malléables. Les reformulations des identités des Kazakhs-Mongols après la chute des économies socialistes et, surtout, comme nous allons maintenant l'examiner, lors des mouvements migratoires au Kazakhstan, témoignent de leur appartenance à un groupe caractérisé par l'attachement à une culture kazakhe et à un territoire mongol. En Mongolie, cette sous-culture est par ailleurs bien identifiée par les Mongols.

\section{LES ORALMAN MONGOLS}

Entre 1990 et 2000, les Oralman mongols constituaient le groupe de « rapatriés » le plus important au Kazakhstan, environ 35 \% des quotas officiels (Diener, 2009 : 234). Ils sont depuis largement dépassés par les Kazakhs d'Ouzbékistan, qui représentaient $72 \%$ de l'ensemble des Oralman en 2006 (UNDP, 2006 : 13). La situation des Oralman mongols est, à plus d'un titre, intéressante. Par exemple, le cloisonnement linguistique des Oralman mongols est différent de celui des autres Oralman non-russophones : Chinois, Afghans, Iraniens, Pakistanais ou Turcs. Les Oralman mongols parlent mieux le kazakh que les Kazakhs kazakhstanais, à l'instar des Oralman chinois ; mais, à la différence de ces derniers, ils connaissent et utilisent le cyrillique, adapté aux langues mongole et kazakhe dans les années 1930. Or, les Oralman non-russophones connaissent les plus grandes difficultés à s'insérer au Kazakhstan ${ }^{15}$; la situation atypique de ceux de Mongolie favorise leur marginalité.

L'immigration des Kazakhs de Mongolie au Kazakhstan après 1990 distingue plusieurs « courants » : la migration de cadres et OQ du secteur minier entre 1991 et 1994 ; le regroupement familial entre 1995 et 1999 ; des migrations internes et le développement d'une communauté transnationale entre 2000 et 2005. Depuis, une nouvelle forme de migration semble apparaître grâce au développement des échanges transfrontaliers, nous $\mathrm{y}$ reviendrons dans la troisième partie de cet article. Invités à se rapatrier au Kazakhstan dans le cadre de contrats quinquennaux dans le secteur agricole, les cadres et OQ kazakhs ont connu une véritable dépréciation statutaire. La plupart d'entre eux connaissait peu l'élevage.

14 Selon Becker dont l'ouvrage Outsiders (1968) est précurseur des études en sociologie de la déviance, une « sous culture » (subculture en anglais) caractérise les communautés rassemblées autour de pratiques « déviantes » et les groupes marginalisés. En Mongolie, l'Islam peut conduire à des pratiques considérées comme déviantes et l'appartenance à la nationalité kazakhe entraîne une stigmatisation importante dans certaines activités politiques et économiques.

15 Tous les rapports concernant les Oralman soulignent l'augmentation des difficultés pour ceux d'entre eux qui ne connaissent pas la langue russe et viennent d'un état hors de l'ex-URSS, car le russe est aujourd'hui la seconde langue vernaculaire du Kazakhstan, parfois la première dans le nord du pays. 
Dès qu'ils l'ont pu, parfois avant la fin de leur contrat, les premiers Oralman mongols ont quitté le secteur primaire. En l'absence de connaissance du russe, ils ont connu un fort taux de chômage. Leur insertion a souvent été problématique dans un pays en crise. Entre $35 \%$ et $70 \%$ des Oralman en âge de travailler seraient au chômage en 2005 (Diener, 2009 : 267), tandis que le nombre national de chômeurs n'excèderait pas $10 \%$ de la population du Kazakhstan, 8,4 \% en 2004 selon l'UNDP (2006 : 15). La deuxième vague de migrants profita du regroupement familial. Elle vient trouver un confort inexistant et des infrastructures publiques déliquescentes en Mongolie. Ainsi sont arrivés des anciens qui souhaitaient mourir sur leur « terre ancestrale » et des enfants rapatriés pour étudier (Diener, 2009 : 266).

Généralement, les Oralman ont été installés dans des régions désertées par d'anciennes « victimes diasporiques » (victim diasporas) de l'ex-URSS (Diener, 2009 : 218). À la suite d'un regroupement familial, la plupart des premiers Oralman a quitté la région initiale de son rapatriement au Kazakhstan pour des raisons de correspondance avec son environnement d'origine et afin de lutter contre l'éparpillement démographique (Ibid : 232-235). Les Oralman mongols se sont regroupés dans l'Est du pays et la région d'Almaty, dans les régions montagneuses de l'Altaï. Se rapprochant des parents restés en Mongolie, ils reconstruisent une sous-culture kazakhe mongole dans un environnement comparable à l'écosystème de Mongolie occidentale. Si un petit nombre d'Oralman mongols s'est effectivement reconverti dans l'industrie minière, la plupart a développé des activités de vente de produits « ethniques » (broderies) ou régionaux (viande ${ }^{16}$ ). Certains servent également d'intermédiaires dans l'approvisionnement du négoce transfrontalier des Kazakhs de Mongolie.

Depuis les années 1990, le rapatriement des « diasporas kazakhes » au Kazakhstan vise l'augmentation du nombre de Kazakhs parmi les citoyens kazakhstanais. Beaucoup de ces rapatriés, surtout parmi ceux issus des pays extérieurs à l'ex-URSS, parlaient encore le kazakh et avaient conservé des savoirs et savoir-faire traditionnels liés au nomadisme pastoral (cuisine, broderie, organisation sociale, etc.) souvent oubliés au Kazakhstan. L'arrivée massive d'Oralman au Kazakhstan entraîna donc un processus de redéfinition de l'« identité kazakhe ». Dans le contexte d'une crise économique profonde et de la restructuration post-socialiste, ce processus de (re)définition identitaire s'est rapidement transformé en sentiment nationaliste et xénophobe.

\section{DES COMMUNAUTÉS EN MOUVEMENT}

Le contexte politique, social et économique n'a pas favorisé l'intégration des Oralman au Kazakhstan. Beaucoup d'Oralman mongols se sont sentis isolés et marginalisés. Ils ont souffert d'une image péjorative attribuée aux " Mongols » par les Kazakhstanais qui les jugent « feignants », " pingres » et « rustres ». Ceux qui sont finalement retournés en Mongolie évoquent leurs difficultés à vivre au Kazakhstan, leur assi-

16 L'élevage extensif reste pratiqué en Mongolie. Ce pays produit et exporte de grandes quantités de viande. En 2006, Bajan-ölgij possédait un abattoir dont les pratiques se conformaient aux règles de l'Islam. La viande débitée était majoritairement exportée vers la Russie et le Kazakhstan. 
milation aux Mongols sur leur «terre ancestrale », les discriminations dont ils ont fait l'objet face à l'émergence du nationalisme kazakhstanais. Ceux qui ont fait le choix de rester n'ont guère demandé la nationalité kazakhe, car ils préfèrent continuer à bénéficier des aides et des allocations attribuées aux « rapatriés » par le gouvernement kazakh ou de celles octroyées à ses ressortissants par le gouvernement mongol.

Les premiers Oralman mongols ont connu de nombreuses difficultés socioéconomiques. La venue des membres de leur famille lors de la seconde vague de migration a favorisé leur reconversion dans les secteurs du commerce transfrontalier et du petit négoce. En effet, en sortant de leur solitude et de leur isolement, les premiers Oralman mongols ont pu ouvrir des comptoirs de vente en Mongolie, à Bajan-ölgij, avec des produits importés du Kazakhstan ou au Kazakhstan avec des produits de Mongolie ou de Chine. Pour ce faire, la citoyenneté mongole est indispensable car les Mongols n'ont pas besoin de visa pour voyager dans les régions frontalières de la Russie et de la Chine.

Depuis les années 2000, les Oralman mongols se spécialisent dans des activités transnationales (Diener, 2009 : 335). Nombre de mes informateurs kazakhs de BajanÖlgij ont développé des stratégies commerciales transnationales entre la Mongolie, la Russie, le Kazakhstan et la Chine. Lors de l'enquête de terrain que j'ai conduite dans cette province de Mongolie en 2002, j'ai rencontré beaucoup de négociants transfrontaliers qui tenaient, en famille, des étals sur les marchés. Durant l'hiver, l'époux parcourait les routes de Russie et de Chine pour approvisionner les étals tenus par les femmes sur les marchés mongols ou russes. Grâce à la collaboration des membres de leur famille, certains avaient ouvert deux étals, l'un en Mongolie et l'autre dans la province russe de Gorno-Altaj.

En 2006, les plus riches de ces négociants avaient élargi leur réseau d'activités grâce à des membres de leur famille « rapatriés » au Kazakhstan. Certains déployaient des « stratégies de migration temporaire ». Au sein d'un même lignage, des parents migrent au Kazakhstan et s'enregistrent comme Oralman. Une fois épuisé le système d'aides destinées aux Oralman, ils rentrent en Mongolie et un autre membre du lignage prend leur place. Cette stratégie permet de profiter du système d'aide au rapatriement sans perdre le bénéfice des investissements effectués localement en Mongolie et au Kazakhstan. Ces «migrations alternées » sont planifiées entre les membres d'un même lignage qui développe ainsi une stratégie collective pour déployer leurs activités commerciales transfrontalières tout en bénéficiant des aides qui leur sont attribuées. Ces stratégies pourraient expliquer l'excès de 30000 personnes dans le décompte des Kazakhs-Mongols de Mongolie et du Kazakhstan.

Depuis 2000, une tendance nouvelle se repère dans la pérennisation des stratégies transnationales des Oralman mongols. En Mongolie et au Kazakhstan, les KazakhsMongols entretiennent aujourd'hui des liens « ethnico-territoriaux » en utilisant - voire en les (re)construisant - leurs réseaux de parenté présents en Chine et en Russie. Ils se distinguent par une « stratégie de mouvements », privilégiant les relations transnationales, le commerce transfrontalier et la mobilité entre différents lieux au sein d'un réseau de parenté. Ils confirment, par conséquent, l'existence d'une sous-culture spécifique à leur communauté. 


\section{ÉPILOGUE}

On ne peut conclure, même temporairement, concernant les recompositions identitaires des Kazakhs-Mongols, car ils construisent et reconstruisent en permanence de nouvelles stratégies communautaires. Nous proposons un épilogue à cette analyse dans la comparaison avec certaines des stratégies migratoires des Mongols de Mongolie. À l'instar du Rapport sur le développement humain en Mongolie (UNDP, 2003 : 43), il faut déplorer l'absence de travaux publiés sur les migrations actuelles des Mongols. La Corée du Sud, le Kazakhstan et la République tchèque constituent leurs destinations de prédilection. On peut interroger cette absence. Provient-elle du faible nombre de personnes concernées par ces migrations ? De la difficulté de leur investigation, les locuteurs de mongol s'intéressant à des problématiques plus locales que mondialisées ?

Si la presse mongole évoque souvent la question des migrations, c'est pour mettre en évidence les difficultés et les situations problématiques vécues par certains migrants mongols. Le regard de la presse locale est biaisé par un sentiment nationaliste qui déprécie les migrants, considérés comme ayant « fui » les difficultés du pays. La question des migrations contemporaines des Mongols préoccupe les spécialistes occidentaux de la Mongolie, car elle se trouve au cœur de l'actualité mongole ; mais personne n'a encore tenté d'en faire une analyse exhaustive. En revanche, les scientifiques asiatiques, en particulier les Coréens et les Japonais, sont à l'œuvre et tout laisse à croire que différents travaux sont en cours de publication.

Depuis la restructuration post-socialiste des années 1990, les Mongols ont utilisé leur situation « $\operatorname{tampon}^{17}$ », et les privilèges qui y sont liés, en particulier, la relative simplicité des formalités administratives nécessaires pour séjourner en République Fédérale de Russie, dans quelques pays de la CEI ou en RPC. Par exemple, entre 1991 et 2001, ce sont en majorité des Mongols qui ont approvisionné la Russie en produits manufacturés chinois car ils n'avaient pas besoin de visa pour aller en Chine et en Russie ${ }^{18}$. Les « années d'or » $\mathrm{du}$ « négoce à la valise » $\left(\right.$ najma $\left.^{19}\right)$ ont ouvert des voies commerciales jusqu'en Europe de l'Est. Les Mongols se sont, en effet, brièvement spécialisés dans le convoi de voitures « de seconde main », achetées sur le marché allemand et revendues en Mongolie. Les « routes transnationales » de la Mongolie vers certains de ses voisins voient toujours défiler un nombre important de négociants mongols. Leurs trajets s'arrêtent aujourd'hui aux régions frontalières, car les formalités de séjour libre ont été limitées par des accords bilatéraux conclus au début des années 2000 entre la Mongolie et ses deux voisins, la Chine et la Russie (Lacaze, 2010).

À la différence des Kazakhs, les Mongols n'ont pas l'opportunité de migrer de manière pérenne dans un autre État voisin. Ni la Mongolie, ni la Chine, pas plus que la

17 La Mongolie occupe la place d'État tampon entre la Russie et la Chine.

18 Soulignons l'assimilation des négociants mongols à des Chinois dans l'ouvrage de Tinguy (2004: 409-445).

19 Le terme mongol najmaa dérive du terme chinois maimai qui signifie : le négoce, le commerce. En mongol, le najmaa désigne en particulier le «négoce à la valise » caractéristique de la période de restructuration post-socialiste. 
Russie n'entretiennent les aspirations pan-mongolistes émergentes depuis 1990. Aucun de ces États n'a appelé les Mongols au rapatriement. Les Mongols, néanmoins, migrent de manière temporaire dans les régions frontalières de la Chine ou de la Russie où ils y jouissent d'un droit de séjour limité dans le temps. Ainsi, on compte plusieurs milliers de migrants «temporaires permanents » à Erlian-Ereen, ville située sur la ligne ferroviaire du trans-mongol, à la frontière chinoise. Ces Mongols sont des migrants temporaires, car ils n'ont qu'un droit de séjour de trente jours à la frontière sino-mongole. Ils résident néanmoins à Ereen de manière permanente en sortant de Chine une fois par mois. Ils font mensuellement un aller-retour au poste frontière de Zamyn-üüd afin de renouveler leur droit de séjour à Ereen, en Chine (Lacaze, 2010).

Il faut, pour finir, mentionner les migrations mongoles dans les pays « riches ». Les destinations de prédilection des Mongols sont la Corée du Sud, l'Europe, le Japon et les États-Unis. N'ayant pas d'informations précises sur ces deux derniers pays, nous n'évoquerons que la Corée du Sud et l'Europe. Ces deux exemples illustreront la diversité des trajectoires migratoires actuelles des Mongols. À la fin des années 1990, la Corée du Sud offrait une destination migratoire appréciée des Mongols. En 2002, près de 20000 ressortissants travaillaient illégalement dans les secteurs de la construction et des services. Depuis, plusieurs accords bilatéraux visent à régulariser les Mongols de Corée. Ainsi, en 2008, les gouvernements des deux États créent une assurance médicale spéciale pour les immigrés. Le gouvernement coréen a organisé plusieurs campagnes de régularisation et favorisé les contrats de travail destinés aux Mongols. En revanche, depuis 2008 et la crise internationale, il tente de réduire les migrations de travail et freine, en particulier, l'arrivée de nouveaux migrants en provenance de Mongolie. En 2010, la situation des Mongols en Corée du Sud est mieux encadrée qu'auparavant, tandis que le nombre de nouveaux immigrants est régulé.

Comparativement à la Corée, les Mongols sont peu nombreux en Europe. Il y en aurait plusieurs milliers, mais il reste difficile de connaître leur nombre exact, car la majorité y réside illégalement. Les migrations des Mongols vers l'Europe sont essentiellement temporaires et, essentiellement, à des fins de formation ou de coopération. Parmi les résidents permanents, beaucoup de Mongol-es sont marié-es à des ressortissants européens. D'autres ont bénéficié d'accords universitaires et sont demeurés dans leur pays d'accueil une fois finies leurs études. C'est, par exemple, le cas de la majorité des membres des communautés mongoles d'Allemagne et de République tchèque ${ }^{20}$ arrivée durant les années 1990. Ces deux communautés jouent, aujourd'hui, le rôle d'intermédiaire dans les réseaux qui organisent les nouvelles migrations des Mongols sur le territoire de l'Union européenne. Ainsi, la plupart des résidents mongols illégaux sur le territoire de l'UE a emprunté la route qui mène en bus jusqu'à Prague ${ }^{21}$, avant de se rendre quelque temps en Allemagne. De là, les migrants se dirigent vers d'autres pays, principalement, la France,

$20 \mathrm{Au}$ sein de l'UE, la récession a particulièrement touché les populations migrantes en 2008-2009. Plusieurs pays ont proposé des « aides aux retours 》. En République tchèque, près de 5000 personnes en ont bénéficié, principalement des Mongols et des Vietnamiens.

21 En 2008, le trafic de « visa tchèque » faisait encore florès sur le marché noir d'Ulaanbaatar et dans l'organisation d'arnaques liées à l'émigration illégale au départ de la Mongolie. Alors même que la République avait adhéré à l'espace Schengen! 
la Belgique 22 ou les Pays-Bas, ainsi que la Suède et le Danemark. Ils essaient également, via l'espace Schengen, d'atteindre la Suisse et le Royaume-Uni.

Les citoyens mongols seraient moins d'un millier à vivre en France : une centaine d'étudiants accueillie chaque année, une centaine de Mongol-es marié-es à des ressortissants français et plusieurs centaines de migrants illégaux. Ces derniers sont restés en France après l'expiration de leur visa ; rares sont ceux entrés illégalement sur le territoire. Les quelques expertises, traductions et séances d'interprétariat que j'ai effectuées auprès de migrants mongols résidant de manière illégale en France indiquent qu'une partie d'entre eux vit en volant dans des magasins de luxe. Un réseau « maffieux » s'est structuré autour de cette activité : volés sur le territoire français, les produits de luxe - essentiellement vêtements et parfums - sont revendus sur le marché néerlandais. Pour se prémunir de la répression administrative et policière en France et afin de palier à la crise du logement qui y sévit, les Mongols illégaux résident en Belgique. Le réseau assure quotidiennement le transport des personnes de la Belgique vers la France et le transport des marchandises de la France vers les Pays-Bas. Cette activité fait aujourd'hui florès à tel point qu'en Mongolie, les Mongols de France sont réputés pour être des « voleurs ».

Certains des Mongols qui résident de manière illégale en Europe développent donc un réseau « ethnique » de commerce transnational. Ils assurent l'approvisionnement, le transport et la vente des marchandises, en l'occurrence des produits volés à l'industrie du luxe. À chaque étape du parcours d'acheminement des produits, une communauté des ressortissants mongols sert d'intermédiaire. Cette activité répréhensible s'organise de manière comparable au commerce transfrontalier des Kazakhs-Mongols, quant à lui, plus ou moins légal. Elle renvoie plus largement au commerce transfrontalier développé par les citoyens mongols (Lacaze, 2008). Ainsi, quelques Mongols et Kazakhs de Mongolie se spécialisent dans des activités comparables de commerce itinérant et transfrontalier. Développé et répandu dans les anciens pays socialistes, le " commerce à la valise », ethnique et transfrontalier, devient une véritable « spécialité locale » des citoyens mongols qui bénéficient de droits privilégiés de séjour et d'entrée en Chine et en Russie. Ces derniers utilisent leur réseau élargi de parents, en les reconstruisant ou les réinventant, et profitent ainsi de la dispersion géographique des peuples mongols. Les Kazakhs-Mongols semblent développer les mêmes stratégies.

22 En 2008, 119 citoyens de Mongolie ont bénéficié de l'« aide au retour » accordée par le gouvernement belge, tandis que onze d'entre eux étaient expulsés de force (Réseau Européen des Migrations, 2009 : 18). Plus d'une centaine de ressortissants mongols sont arrêtés en Belgique chaque année depuis 2005. Aucun des ressortissants mongols n'a fait de demande d'asile en Belgique (ibid. : 13-14). Plusieurs d'entre eux, en revanche, sont connus des bureaux français de l'OFPRA. 


\section{Références bibliographiques}

ANN (2008) Mongolie, http://www.tlfq.ulaval.ca/AXL/asie/Mongolie.htm, consulté le 03/11/2010. BECKER Howard S. (1968) Outsiders : études de la sociologie de la déviance, Paris, Métailié, $247 \mathrm{p}$.

BULAC Uradyn (1998) Nationalism and hybridity in Mongolia, Oxford, Clarendon Press, 272 p.

DIENER Alexander C. (2009) One homeland or Two? The Nationalization and Transnationalization of Mongolia's Kazakhs, Chicago, Stanford University Press, 405 p.

EVEN Marie-Dominique et POP Rodica (trad.) (1994) Histoire secrète des Mongols (Mongghol-un ni'uca tobciyan) Chronique mongole du XIIIe siècle, Paris, Gallimard, 349 p.

EVEN Marie-Dominique (1996) Un entretien avec Dj. Enkhsaïkhan sur la politique extérieure de la Mongolie, Anda, 20, http://www.anda-mongolie.com/propos/politique/enkhsai20.html, consulté le $03 / 11 / 2010$.

FIDH (2009) Kazakhstan/Kirghizstan: Exploitation of Migrants Workers, Protection Denied to Asylum Seekers and Refugees, Rapport n530a Oct 2009, Paris, 89 p.

LACAZE Gaëlle (2008) Mise en rythme d'une esthétique du pouvoir. Les perceptions esthétiques dans le contexte mongol et sibérien, Actes de la Journée d'étude "Que sont les perceptions esthétiques dans un contexte mongol et sibérien » (20/10/07), Réseau-Asie-CEMS, Paris, http:// www.reseau-asie.com/congrès/\%20Journée\%20d'études/document $\% 20555$.

LACAZE Gaëlle (2010) "Run after time": the roads of suitcase traders, Asian ethnicity, 11 (2), pp. 191-208.

RÉSEAU EUROPÉEN DES MIGRATIONS. Point de contact belge (2009), Programmes et stratégies en Belgique pour le retour volontaire assisté et la réintégration dans un pays tiers, Bruxelles, $37 \mathrm{p}$.

TINGUY Anne (de) (2004) La grande migration : la Russie et les Russes depuis l'ouverture du rideau de fer, Paris, Plon, $662 \mathrm{p}$.

UNDP (2006) Status of Oralmans in Kazakhstan. Overview, Almaty, UNDP, 31 p.

UNDP (2003) Human Development Report. Mongolia, Ulaanbaatar, UNDP, 79 p. 